\title{
Análise estrutural de viga de aço em fase executiva da aplicação de protensão
}

\section{Structural analysis of steel beam in executive phase of the application of}

\section{prestressed}

Article Info:

Article history: Received 2021-03-29 / Accepted 2021-03-29 / Available online 2021-03-30

doi: 10.18540/jcecvl7iss1pp11941-01-09e

\author{
Pedro Ignácio Lima Gadêlha Jardim \\ ORCID: https://orcid.org/0000-0002-4448-6554 \\ Universidade Federal de Rondônia, Rondônia \\ E-mail: eng.pedrojardim@gmail.com \\ Benjamim Nogueira da Silva \\ ORCID: https://orcid.org/0000-0001-6254-6269 \\ Universidade Federal de Rondônia, Rondônia \\ E-mail: benjamim_pvh@hotmail..com \\ Lívia Maria Palácio Ribeiro \\ ORCID: https://orcid.org/0000-0003-2925-8139 \\ Universidade Federal de Rondônia, Rondônia \\ E-mail: livia.ribeiro@unir.br \\ Diego Henrique de Almeida \\ ORCID: http://orcid.org/0000-0002-6220-4004 \\ Universidade Federal de Rondônia, Rondônia \\ E-mail: diegoalmeida@unir.br
}

\begin{abstract}
Resumo
A utilização da protensão como solução para estruturas de aço foi proposta desde a década de 1950 e, apesar de diversos estudos publicados, ainda não está difundida no meio profissional. A sua aplicação é feita de forma externa à estrutura e, entre suas aplicações, é recomendada para reabilitação e reforço. Seu método executivo é relativamente simplificado, uma vez que pouco se altera dos procedimentos executivos das estruturas de aço passivas e do concreto protendido. Este trabalho tem como objetivo analisar o comportamento de uma viga de aço reabilitada quando submetida às etapas de protensão. Para isso, serão aplicadas cordoalhas, causando flexão também no sentido de menor inércia do elemento estrutural. De acordo com o modelo proposto, pode-se observar que os esforços presentes na etapa executiva não superam a capacidade de suporte do elemento. Entretanto, determinadas fases de carregamento podem causar instabilidade caso não sejam verificadas corretamente.
\end{abstract}

Palavras-chave: Estrutura de aço. Protensão. Vigas de aço protendidas.

\begin{abstract}
The use of prestressed as a solution for steel structures has been proposed since the 1950s and, despite several published studies, is not yet widespread in the professional environment. Its application is made externally to the structure and, among its applications, it is recommended for rehabilitation and reinforcement. Its executive method is relatively simplified, since little changes from the executive procedures of passive steel structures and prestressed concrete. This work aims to analyze the behavior of a rehabilitated steel beam when submitted to the stages of prestressed. For this, tendons will be applied, causing bending also in the direction of less inertia of the structural element. According to the proposed model, it can be observed that the efforts present in the executive stage do not exceed the supporting capacity of the element. However, certain loading phases can cause instability if they are not checked correctly.
\end{abstract}

Keywords: Steel structure. Prestressing. Prestressed steel beams. 


\section{Introdução}

$\mathrm{Na}$ engenharia de estruturas existem materiais e tipologias servem de opção para solução de projetos. Para estruturas com grandes vãos e com limitações geométricas, a protensão é uma solução comumente utilizada. Ela tem como objetivo inserir cargas opostas das que estão em serviço no elemento (ALMOHAMADY, 2009). A aplicação mais conhecida é em conjunto ao concreto armado, sendo amplamente utilizado em residências, prédios e pontes. Entretanto, sua utilização não se restringe a esse material.

Uma das opções de aplicação da protensão é adotá-la em conjunto ao aço. O desenvolvimento e estudo das estruturas de aço protendidas teve seu início na década de 1950 com Gustave Magnel. $\mathrm{Na}$ ocasião, ao propor a protensão em treliças, o autor obteve flambagem excessiva no banzo do elemento (MAGNEL, 1950). Na década de 1960, Peter George Hoadley propôs um ábaco de dimensionamento relacionando o vão e a altura da seção. Na década seguinte, os autores Miroslav Tochacek e Champa Lal Mehta desenvolveram um método racional de cálculo para projeto otimizado (SAMPAIO JUNIOR, 1976).

Troitsky (1990) apresenta uma redução no custo de obras utilizando vigas de alma cheia protendidas de $12 \%$ e $30 \%$ para sistemas estruturais em arcos, quando comparado com estruturas de aço sem protensão. Vipparthy e Ponnada (2015) encontraram uma vantagem econômica de $15 \%$.

A utilização da protensão nas vigas de aço não é indicada somente para novas construções, mas também para reabilitação de estruturas existentes (BELLETI; GASPERI, 2010). Para Vipparthy e Ponnada (2015), dentre as possíveis aplicações do método, destaca-se a utilização para reabilitação de estruturas existentes, construções temporárias, reforço estrutural e novas construções.

No Brasil, foi adotada a solução da protensão externa para reforço de vigas de um pavimento garagem do Hotel Hilton, torre leste do Centro Empresarial Nações Unidas, na cidade de São Paulo/SP, em 2002. O projeto foi feito pelo Eng. Mario Franco, com consultoria da Eng. ${ }^{a}$ Heloísa Maringoni. O reforço se deu em virtude da alteração do programa de necessidade dos clientes e, na ocasião, a utilização de concreto protendido resultaria em seções inviáveis para o local (FERREIRA, 2007).

Conforme apontado no estudo de Jardim et al (2020), a aplicação de protensão em um sistema de viga biapoiado confere melhor distribuição das tensões internas no elemento, proporcionando um melhor aproveitamento do material.

Para Almohamady (2009), a falta do conhecimento sobre o método de protensão em elementos de aço e seu método de cálculo é a principal razão para a não utilização do mesmo em obras correntes.

O objetivo desse estudo é verificar o comportamento estrutural de uma viga de aço submetida a protensão em virtude da necessidade de reabilitação da mesma devido a um acréscimo de carregamento. A análise pretende verificar, utilizando o método dos elementos finitos, o estado das tensões internas durante a etapa executiva de tracionamento dos cabos, onde serão inseridos esforços de protensão com dupla excentricidade, resultando em momento fletor no sentido da menor inércia da viga.

\section{Referencial Teórico}

Conforme Almeida (2001), a reabilitação de uma estrutura que não possui capacidade de suporte é a maneira mais adequada para garantir a segurança estrutural ao elemento, sendo o termo "reabilitação" englobando as definições de "reforço" e "recuperação". Segundo Viana (2004), a protensão externa atribui agilidade ao processo de reforço, permitindo que o procedimento seja realizado sem descarregar a estrutura e seu efeito é implementado logo após a conclusão do serviço.

Para o caso da protensão externa, os efeitos da protensão são transmitidos para o elemento estrutural por meio dos desviadores e ancoragens, conforme o traçado do cordoalha (ALMEIDA, 
2001). Conforme Ferreira (2007), o traçado das cordoalhas é de suma importância e define o tipo de comportamento da força de protensão e como será transmitida à estrutura. Para a autora, os traçados mais comuns são os poligonais e, por vezes, os retos. Para Flôr e Amaral (2013), o traçado reto é posicionado abaixo da linha neutra da viga e é indicado para momentos fletores uniformes.

Segundo Nelsen (2013), o traçado poligonal é definido conforme a curvatura do momento solicitante, apresentando maior eficiência para vigas com carregamento distribuído ou com cargas pontuais no tramo do elemento. Entretanto, esse sistema apresenta custo elevado e maior dificuldade de execução, considerando a necessidade da utilização de desviadores para garantir o traçado. Um exemplo desse traçado é visto no estudo de Nunziata (1999) que ensaiou uma viga com 21,40 metros de vão submetida a carregamento distribuído com uma deformação máxima de 79,84 mm (Figura $1)$.

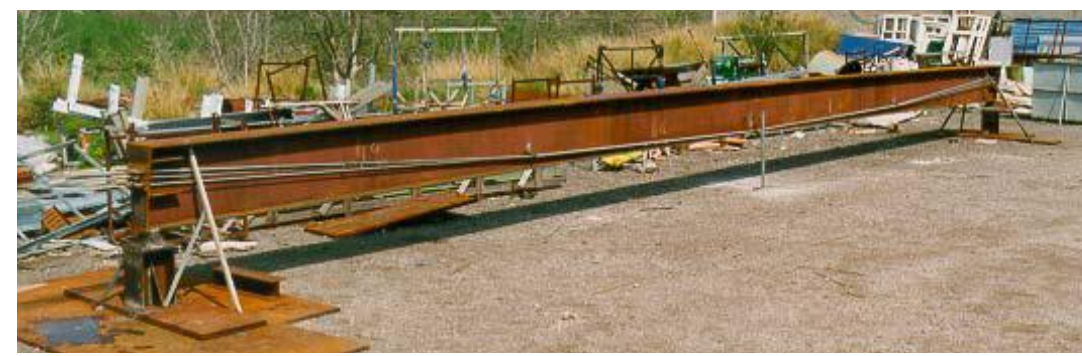

Figura 1 - Viga metálica protendida com traçado poligonal. Fonte: Nunziata (1999)

Para Rezende (2007), a hipótese para o emprego de protensão em vigas metálicas é o controle das flechas. Segundo Ferreira (2007), a interação entre o momento fletor gerado pela protensão e as ações externas são superpostas, resultando um estado de tensões menos crítico, apesar de conferir um esforço axial adicional que deve ser verificado.

O dimensionamento de elementos de aço protendido é feito de forma análoga ao concreto protendido, sendo que o dimensionamento é realizado inicialmente no estado-limite de serviço (ELS) e posteriormente é verificado no estado-limite último (ELU), baseando a solução nos deslocamentos do sistema ao invés das tensões (REZENDE, 2007).

Para Almohamady (2009) e Gaykar (2013), os métodos mais conhecidos para analisar as seções protendidas são: o método estático, onde os esforços são considerados excêntricos à seção; e o método de cargas equivalentes, onde insere-se um sistema de forças equivalentes às excêntricas.

Uma metodologia de análise estrutural com alto grau de precisão e detalhamento é o Método dos Elementos Finitos (MEF). Segundo Azevedo (2003), o MEF, quando aplicado na Engenharia de Estruturas, consiste na determinação do estado de tensão e de deformação de um sólido com geometria qualquer, quando submetido a ações externas.

A aplicação do método em casos reais consiste na aplicação do procedimento de análise em um sólido espacial, sendo necessário o tratamento das coordenadas dos nós dos subdomínios com um ponto de origem comum (AZEVEDO, 2003). Conforme Vaz (2011), as funções de cada subdomínio devem ser descritas por funções de interpolação.

A análise por elementos finitos é realizada através de um processo de discretização de uma geometria. Este processo consiste em subdividir uma geometria contínua em uma quantidade finita de elementos, ligando-os por nós. Esse conjunto de nós em um elemento é chamado de malha (AZEVEDO, 2016). A aplicação do MEF nas análises de estruturas só se tornou mais comum após a evolução dos computadores para suportarem capacidade de análise e processamento (VAZ, 2011).

\section{Metodologia}

O presente estudo analisou um modelo de viga biapoiada, perfil W 530 x 74, com 10 metros de vão. $\mathrm{O}$ valor do vão foi escolhido por representar uma dimensão usual para fachadas de edificações e o perfil foi determinado em função do mesmo. A viga, inicialmente dimensionada para 
um carregamento distribuído característico permanente (Gk) de $14,40 \mathrm{kN} / \mathrm{m}$ (proveniente de laje maciça de 8 x 10 metros e 20 centímetros de espessura) e acidental (Qk) de $6 \mathrm{kN} / \mathrm{m}$, foi submetida a um carregamento adicional permanente de $3,64 \mathrm{kN} / \mathrm{m}$ e $3,60 \mathrm{kN} / \mathrm{m}$ acidental. Simulando a construção de uma parede de alvenaria sob o elemento e a alteração de utilização do ambiente. Dessa forma, o carregamento permanente total considerado nesta pesquisa foi igual a $18,97 \mathrm{kN} / \mathrm{m}$, já considerando o peso próprio do perfil, e $9,60 \mathrm{kN} / \mathrm{m}$ de carregamento acidental.

A solução proposta para reabilitação da estrutura em virtude das novas cargas foi a utilização de três cabos de protensão com $12,7 \mathrm{~mm}$ de diâmetro. $\mathrm{O}$ traçado utilizado foi o reto, locado abaixo da mesa inferior. Apesar do traçado não ser o mais eficiente quanto à análise estrutural, essa escolha se deu em virtude da facilidade da instalação e execução da protensão, considerando que esse estudo simula uma estrutura já executada. Para as simulações, foram considerados que os cabos seriam fixados a 329,50 mm abaixo do centro de gravidade (CG) do perfil, com um cabo central e dois com equidistância de 63,5 mm do eixo vertical da seção do perfil (Figura 2).

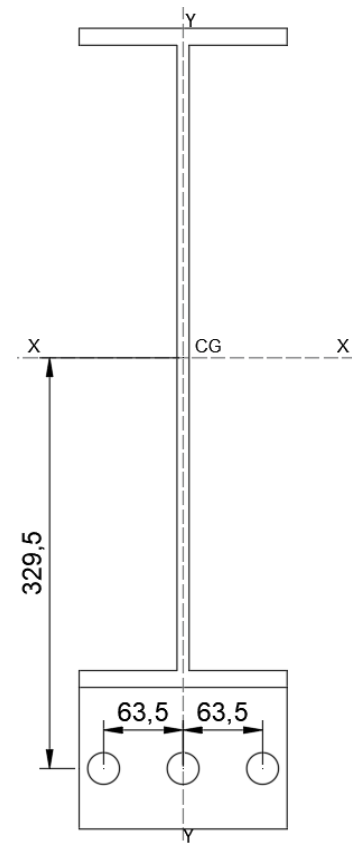

Figura 2 - Seção transversal da peça estudada (medidas em mm)

Inicialmente foi verificado os efeitos da protensão no ELS e ELU de forma analítica, objetivando avaliar se o sistema proposto atende os quesitos de estabilidade. Posteriormente, foi realizada a análise computacional para avaliação das tensões internas e verificação quanto a melhoria do aproveitamento do elemento estrutural para o caso inicial de carregamento e para o caso final onde são considerados os efeitos da protensão e o carregamento adicional.

A análise computacional foi feita por meio do software Ansys ${ }^{\circledR}$, onde a viga foi modelada e analisada quanto sua deformação total e distribuição das tensões internas tridimensionais. A viga foi modelada com 23068 nós, modelada utilizando o elemento solid, com aço estrutural A36. Para tal, as fases de carregamento foram divididas em cinco casos, considerando as etapas de aplicação de carregamento no elemento estrutural reabilitado, descritos pela Tabela 1.

Tabela 1 - Casos de carregamento

\begin{tabular}{|c|c|c|}
\hline Casos & Descrição & Carregamento / kN; m \\
\hline 1 & Sistema inicial & $\begin{array}{l}\mathrm{G}_{\mathrm{k}}=14,40 ; \mathrm{Q}_{\mathrm{k}}=6,00 \\
\mathrm{~N}_{\mathrm{p}}=\mathrm{M}_{\mathrm{x}}=\mathrm{M}_{\mathrm{y}}=0,00\end{array}$ \\
\hline 2 & Introdução da cordoalha central & $\begin{array}{l}\mathrm{G}_{\mathrm{k}}=14,40 ; \mathrm{Q}_{\mathrm{k}}=6,00 \\
\mathrm{~N}_{\mathrm{p}}=140,85 ; \mathrm{M}_{\mathrm{x}}=46,41 \\
\mathrm{M}_{\mathrm{y}}=0,00\end{array}$ \\
\hline
\end{tabular}




\begin{tabular}{cll}
\hline \multirow{2}{*}{3} & & $\mathrm{G}_{\mathrm{k}}=14,40 ; \mathrm{Q}_{\mathrm{k}}=6,00$ \\
& Introdução da cordoalha central e uma lateral & $\mathrm{N}_{\mathrm{p}}=281,70 ; \mathrm{M}_{\mathrm{x}}=92,82$ \\
& $\mathrm{M}_{\mathrm{y}}=8,94$ \\
\hline \multirow{3}{*}{ Introdução de todas as cordoalhas } & $\mathrm{G}_{\mathrm{k}}=14,40 ; \mathrm{Q}_{\mathrm{k}}=6,00$ \\
& & $\mathrm{~N}_{\mathrm{p}}=422,55 ; \mathrm{M}_{\mathrm{x}}=139,23$ \\
& $\mathrm{M}_{\mathrm{y}}=0,00$ \\
\hline \multirow{2}{*}{5} & Sistema final, com todas as cordoalhas e & $\mathrm{G}_{\mathrm{k}}=18,97 ; \mathrm{Q}_{\mathrm{k}}=9,60$ \\
& carregamento adicional & $\mathrm{N}_{\mathrm{p}}=422,55 ; \mathrm{M}_{\mathrm{x}}=139,23$ \\
& $\mathrm{M}_{\mathrm{y}}=0,00$ \\
\hline
\end{tabular}

Nota: A viga foi modelada com as seguintes propriedades mecânicas: $\mathrm{E}=200 \mathrm{GPa}, \mathrm{f}_{\mathrm{y}}=250 \mathrm{MPa}$ e $\mathrm{f}_{\mathrm{u}}=400 \mathrm{MPa}$.

Os carregamentos descritos na Tabela 1 são característicos, sendo adotado para os cálculos e verificações os seguintes valores de combinações: $29,37 \mathrm{kN} / \mathrm{m}$ para combinação normal no estado limite último (ELU) e 17,54 kN/m para combinação quase-permanente no estado limite de serviço (ELS); os esforços de protensão permanecem os mesmos no ELS e são majorados em $20 \%$ no ELU.

A verificação do desempenho da viga no ELU foi feita de forma isolada para os esforços normais de compressão e momento fletor, verificando se a peça ainda apresenta capacidade portante para cada caso. Posteriormente, foi realizada a verificação da interação desses esforços, analisandoos combinadamente, devendo seu valor estar sempre abaixo de 1 . O método utilizado é apresentado por Souza (2018).

Foram avaliadas as deformações do elemento para cada caso e a influência dos efeitos de protensão durante a instalação de cada cordoalha. Os limites de deslocamento foram determinados conforme ABNT NBR 8800 (2008), sendo o vão da viga divido por 350 para casos sem alvenaria sob a viga (casos 1 até 4) e, para o Caso 5, o limite é definido pela fissuração da alvenaria (15mm). No Caso 5, foi avaliada a eficiência do sistema estrutural e o aproveitamento dos materiais empregados.

A avaliação dos resultados obtidos foi realizada relacionando-os com os respectivos valores máximos de resistência e de deslocamento para cada caso, sendo esta relação expressada em porcentagem. Para valores próximos de $100 \%$, a peça apresenta melhor aproveitamento de sua capacidade portante e, por tanto, é mais econômica.

\section{Resultados}

A Tabela 2 apresenta os valores máximos obtidos para o deslocamento no centro do vão em cada caso de carregamento e protensão simulados nessa pesquisa, bem como o deslocamento limite considerando o ELS.

Tabela 2 - Deslocamentos da viga para cada caso de carregamento

\begin{tabular}{ccc}
\hline Casos & Deslocamento encontrado / cm & Deslocamento limite / cm \\
\hline 1 & 2,79 & 2,86 \\
\hline 2 & 2,08 & 2,86 \\
\hline 3 & 1,37 & 2,86 \\
\hline 4 & 0,66 & 2,86 \\
\hline 5 & 1,50 & 1,50 \\
\hline
\end{tabular}

As verificações no ELU foram realizadas inicialmente pela análise da resistência axial do perfil, sendo sucedida pela averiguação da resistência à flexão em cada caso e finalizada com a verificação de interação devido flexão composta. Os valores dos esforços solicitantes estão relacionados na Tabela 3 . 
Tabela 3 - Esforços solicitantes no ELU

\begin{tabular}{cccc}
\hline Casos & $\begin{array}{c}\text { Normal solicitante / } \\
\text { kN }\end{array}$ & $\begin{array}{c}\text { Momento fletor máximo / } \\
\text { kN.m }\end{array}$ & $\begin{array}{c}\text { Verificação de } \\
\text { interação }\end{array}$ \\
\hline 1 & 0,00 & 367,13 & - \\
\hline 2 & 169,02 & 311,43 & 0,76 \\
\hline 3 & 338,04 & 255,74 & 0,94 \\
\hline 4 & 507,06 & 200,05 & 0,62 \\
\hline 5 & 507,06 & 338,67 & 0,96 \\
\hline
\end{tabular}

A modelagem do Caso 1 e do Caso 5 é apresentada na Figura 3. Devido sua simetria, estão ilustrados os mapas de tensão da metade do elemento. À esquerda é a região do apoio e à direita o meio do vão. Na Figura 3, é ilustrada a viga com o carregamento inicial (Figura 3a) e após a aplicação da protensão e carregamento adicional (Figura 3b).
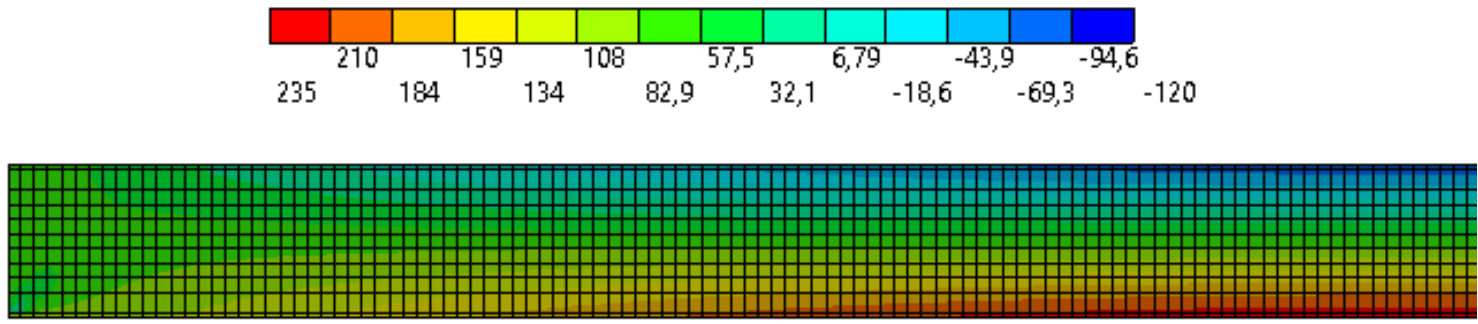

(a)

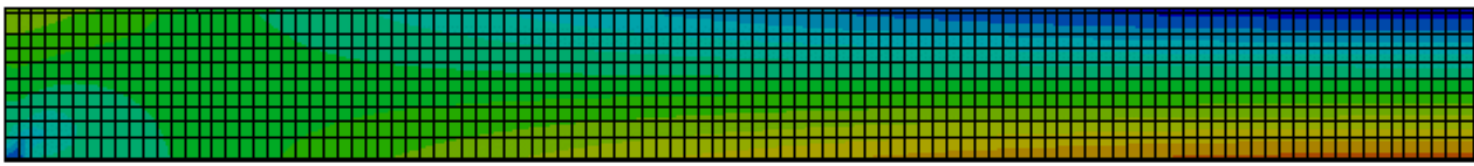

(b)

Figura 3 - Distribuição interna das tensões de flexão para o Caso 1 (a) e Caso 5 (b) (MPa)

\section{Discussões}

A verificação de cada etapa da aplicação da protensão com função de reabilitação de uma estrutura existe é de fundamental importância para que se possa garantir que a solução seja viável, exequível e eficiente. Antes do carregamento adicional, a estrutura está submetida aos esforços de protensão que devem ser avaliados para que não excedam os limites aceitáveis de resistência do elemento de aço. Os resultados obtidos anteriormente são relacionados na Figura 4 apresentando a eficiência do perfil a cada solicitação e discutida posteriormente. 


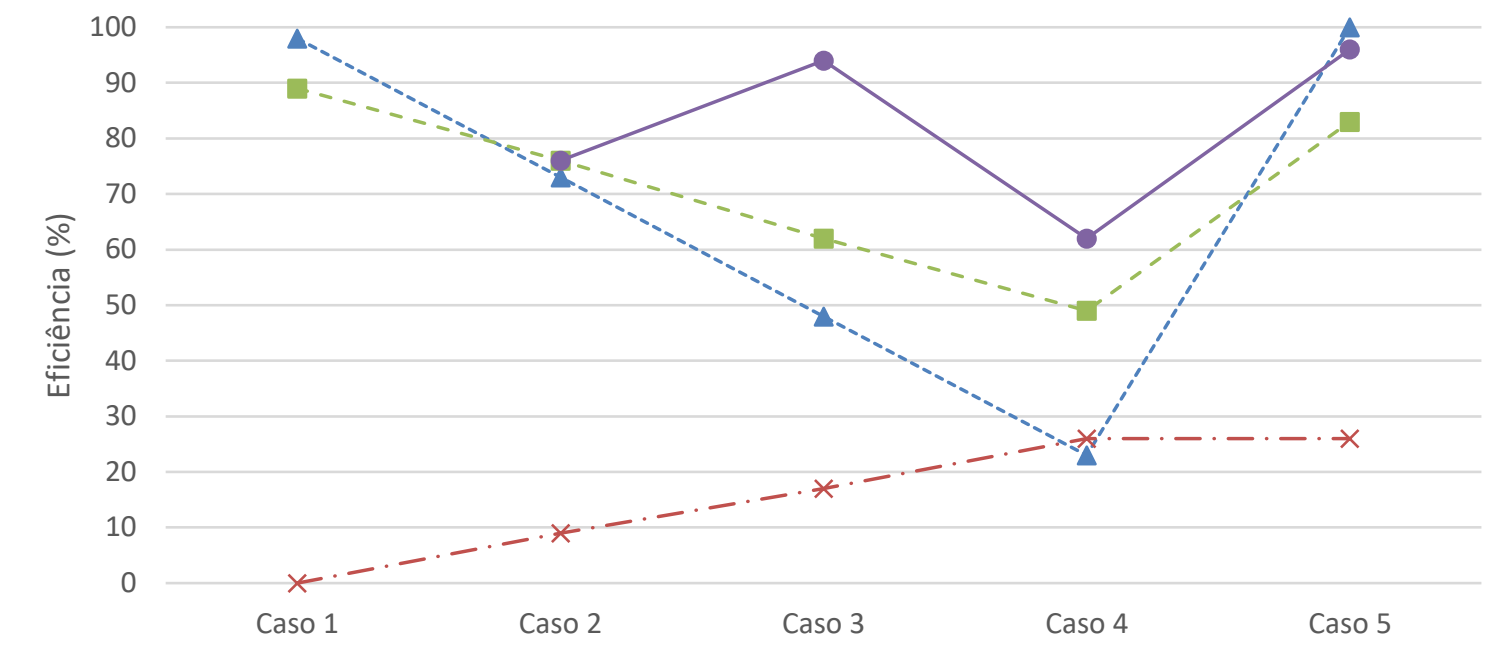

----A--- Deslocamento $-\rtimes-$ Esforço normal de compressão - - Momento fletor - — Interação dos esforços

\section{Figura 4 - Eficiência do perfil no ELS e ELU para cada Caso estudado}

A viga, em seu estado inicial, descrita pelo Caso 1, apresenta boa eficiência quanto à sua resistência, com um aproveitamento de $89 \%$ da capacidade portante do elemento. Para a avaliação quanto ao seu deslocamento, a mesma possui $98 \%$ de eficiência, atendendo aos limites de deslocamento devido aceitabilidade sensorial de forma efetiva e econômica.

A introdução dos carregamentos devidos à protensão, descritos pelos Casos 2, 3 e 4, atribuem esforços de compressão e flexão com sentidos opostos aos demais carregamentos externos. Para o Caso 2, onde apenas a cordoalha central é considerada, o sistema é aliviado e inicia-se a verificação da capacidade portante do elemento quanto à compressão. O deslocamento máximo do elemento para o caso seria de $2,08 \mathrm{~cm}$, apresentando uma redução de $71 \mathrm{~mm}$ em relação ao caso anterior. A compressão inserida no sistema representaria, aproximadamente, apenas $9 \%$ da capacidade da viga e a resistência à flexão teria sua eficiência reduzida para $76 \%$. A verificação quanto a flexão composta, devido a esses carregamentos da protensão, resultam em um aproveitamento de $76 \%$ da capacidade do perfil.

Para o Caso 3, onde o elemento está sujeito à flexão composta oblíqua, devido ao acréscimo da segunda cordoalha, foi observado que o aproveitamento da viga se mantém diminuindo em razão similar aos demais casos estudados: deformação com 48\%, resistência à compressão com $17 \%$ e à flexão com $62 \%$. Entretanto, ao avaliar os esforços com interação, obtemos um resultado de 0,94 , muito próximo ao limite (valor limite de interação é 1). Tal ponto poderia indicar a limitação da peça para as ações da protensão durante sua fase executiva, considerando que ainda restam cordoalhas a serem instaladas.

Entretanto, avaliando os resultados para o Caso 4, com as três cordoalhas participando do sistema, observa-se a mesma razão de decréscimo para o aproveitamento da viga, isoladamente (23\% para deformação, $26 \%$ para compressão e $49 \%$ para flexão), mas a eficiência do perfil quanto a interação dessas solicitações reduziu para 0,62 . Tal fato se deve pela mudança na equação de interação, pode a razão entre a solicitação axial e a resistência do perfil supera 0,2 , e, ainda, os efeitos de flexão em y são anulados devido a simetria na locação das cordoalhas.

Apesar de estar submetida a um maior esforço axial no Caso 4, bem como uma maior flexão no eixo $x$, o valor encontrado para a interação desses esforços é o menor encontrado em todos os casos. O esforço axial assume, para esse caso, um importante papel no elemento estrutural para garantir um resultando menor de interação e proporcionando maior capacidade portante.

O Caso 5 insere no sistema o carregamento adicional, bem como a restrição ao deslocamento em função de alvenaria. Como o dimensionamento da protensão é feita com base no ELS, a eficiência do elemento quanto ao seu deslocamento é de $100 \%$, atingindo o valor exato de $1,50 \mathrm{~cm}$. 
Caso o valor limite ainda fosse o de aceitabilidade sensorial $(2,86 \mathrm{~cm})$ a eficiência nessa avaliação seria de $53 \%$.

No ELU, a eficiência do perfil quanto ao esforço axial permanece o mesmo do Caso 4 (26\%), enquanto a flexão aumenta para $83 \%$ devido ao carregamento distribuído adicional. Dessa forma, o valor da verificação de interação aumenta para 0,96 , sendo bastante próximo do limite normativo.

$\mathrm{Na}$ Figura 3b, é ilustrada a mudança de comportamento na região do apoio do Caso 5, onde surgem esforços de flexão na região próxima às mesas do perfil. Observa-se uma redução da flexão na região central do elemento, similar ao demonstrado por Jardim et al (2020). A viga apresenta uma distribuição de esforços ligeiramente mais eficiente na região da alma da viga.

Apesar do valor do momento fletor solicitante do Caso 5 ser próximo ao do Caso 1, é nítida a suavização na sua distribuição, concentrando valores de tensão menores na região mais solicitada. Essa melhor distribuição garante melhor eficiência no aproveitamento da peça como um todo, evitando concentração de tensões elevadas na transição da alma com a mesa da viga.

\section{Conclusões}

O presente artigo apresentou uma análise do comportamento de uma viga de aço quando submetida a protensão, em suas diferentes fases de aplicação. Os esforços adicionais ocasionam em momento fletor no sentido de menor inércia do elemento estrutural.

De acordo com os resultados apresentados, conclui-se que: é possível a utilização da protensão como alternativa para reabilitação, desde que sejam feitas as análise estruturais durante as etapas de protensão; a compressão proveniente da protensão é um esforço importante para o equilíbrio do sistema estrutural durante sua protensão, quando se verifica a interação dos esforços; o elemento estrutural estudado, mesmo quando submetido a flexão composta obliqua, apresenta a estabilidade e resistência adequada para suportar a introdução de cada esforço adicional isoladamente.

Para elementos estruturais de grandes comprimentos, o deslocamento é um fator preponderante na avaliação estrutural. A reabilitação garantiu que a viga tenha comportamento similar ao seu caso inicial, com uma eficiência ainda maior quanto ao seu deslocamento, viabilizando a mudança de utilização do ambiente e, inclusive, a construção de parede de alvenaria sob a viga.

\section{Agradecimentos}

Os autores agradecem à Universidade Federal de Rondônia, em especial à Pró-Reitoria de Pós-Graduação e Pesquisa (PROPesq), ao Departamento de Engenharia Civil (DECIV) e ao Laboratório de Estruturas e Ensaios Mecânicos (LaEEM).

\section{Referências}

ABNT ASSOCIAÇÃO BRASILEIRA DE NORMAS TÉCNICAS. NBR 8800: Projeto de estruturas de aço e de estruturas mistas de aço e concreto em edifícios. Rio de Janeiro, 2008. $237 \mathrm{p}$.

ALMEIDA, T. G. (2001). Reforço de vigas de concreto armado por meio de cabos externos protendidos. Dissertação de Mestrado, Escola de Engenharia de São Carlos, Universidade de São Paulo, São Carlos, SP, Brasil.

ALMOHAMADY, A. (2009). Finite element analysis of prestressed steel beams. Engineering Research Journal: Faculty of Engineering Minoufiya University. 32(2), 233-238. https://dx.doi.org/10.21608/erjm.2009.69421

AZEVEDO, A. F. M. (2003). Método dos Elementos Finitos. Faculdade de Engenharia, Universidade de Portugal.

AZEVEDO, D. F. O. (2016). Análise estrutural com Ansys Workbench. Domingos Flávio de Oliveira Azevedo. 
BELLETTI, B.; GASPERI, A. (2010). Behavior of prestressed steel beams. Journal Of Structure Engineering. 136(9), 1131-1139.

FERREIRA, A. C. (2007). Vigas metálicas protendidas: análise estática, modal e de ruptura do cabo de protensão e complicativo computacional para projetos. Dissertação de Mestrado, Faculdade de Tecnologia, Departamento de Engenharia Civil, Universidade Federal de Brasília, Brasília, DF, Brasil.

FLOR, E. P.; AMARAL, F. L. (2013). Dimensionamento e verificação de estruturas metálicas protendidas. Monografia de Graduação, Setor de Tecnologia, Universidade Federal do Paraná, Curitiba, PR, Brasil.

GAYKAR, V. M. (2013). Prestressed Steel Beam. Monografia de Especialização, Structural Engineering Department, Veermata Jijabai Technological Institute, Mumbai, Índia.

JARDIM, P. I. L. G.; SILVA, B. N.; ALMEIDA, D. H.; RIBEIRO, L. M. P. (2020). Aplicação de protensão em viga metálica por meio de simulação via método dos elementos finitos. Anais do I Congresso Brasileiro Interdisciplinar em Ciência e Tecnologia, Diamantina, Mg, v. 1, n. 1, p. 640-641, 02 set. 2020. https://doi.org/10.29327/121206.1-65

MAGNEL, G; (1950). Prestressed steel structures. The Structural Engineer. Londres, Inglaterra, p. 285-295, nov. 1950.

NELSEN, A. C. H. (2013). Comportamento estrutural de vigas mistas de aço e concreto com protensão externa. Dissertação de Mestrado, Programa de Pós-Graduação em Estruturas e Construção Civil, Universidade Federal de São Carlos, São Carlos, SP, Brasil.

NUNZIATA, V. (1999). Prestressed Steel Structures. Anais do XVII Congresso C.T.A. Napoli, Itália.

REZENDE, C. R. (2007). Análise estrutural de vigas metálicas protendidas. Dissertação de Mestrado, Programa de Pós-Graduação em Engenharia Civil, Centro Tecnológico, Universidade Federal do Espírito Santos, Vitória, ES, Brasil.

SOUZA, A. S. C. (2008). Dimensionamento de elementos e ligações em estruturas de aço. EdUFSCar.

SAMPAIO JUNIOR, C. F. (1976). Dimensionamento econômico de vigas protendidas de aço. Dissertação de Mestrado, Departamento de Estruturas, Escola de Engenharia de São Carlos, Universidade do Estado de São Paulo, São Carlos, SP, Brasil.

TROITSKY, M. S. (1990). Prestressed steel bridges: theory and design. Van Nostrand Reinhold Company.

VAZ, L. E. (2011). Método dos elementos finitos em análise de estruturas. Elsevier.

VIANA, R. D. A. (2004). Programa para dimensionamento de reforço à flexão e ao cisalhamento de vigas de concreto armado. Dissertação de Mestrado, Universidade Federal do Rio de Janeiro, Rio de Janeiro, RJ, Brasil.

VIPPARTHY, R.; PONNADA, M. R. (2015). Prestressed homogenious steel beams strengthened in compression and tension: a hypothetical case study. International Journal of Research in Engineering And Technology, 4(13), 260-266. 